\title{
Transcriptome profiling of a curdlan-producing Agrobacterium reveals conserved regulatory mechanisms of exopolysaccharide biosynthesis
}

\author{
Anne M Ruffing and Rachel Ruizhen Chen
}

\begin{abstract}
Background: The ability to synthesize exopolysaccharides (EPS) is widespread among microorganisms, and microbial EPS play important roles in biofilm formation, pathogen persistence, and applications in the food and medical industries. Although it is well established that EPS synthesis is invariably in response to environmental cues, it remains largely unknown how various environmental signals trigger activation of the biochemical synthesis machinery.

Results: We report here the transcriptome profiling of Agrobacterium sp. ATCC 31749, a microorganism that produces large amounts of a glucose polymer known as curdlan under nitrogen starvation. Transcriptome analysis revealed a nearly 100 -fold upregulation of the curdlan synthesis operon upon transition to nitrogen starvation, thus establishing the prominent role that transcriptional regulation plays in the EPS synthesis. In addition to known mechanisms of EPS regulation such as activation by c-di-GMP, we identify novel mechanisms of regulation in ATCC 31749, including RpoN-independent NtrC regulation and intracellular pH regulation by acidocalcisomes.

Furthermore, we show evidence that curdlan synthesis is also regulated by conserved cell stress responses, including polyphosphate accumulation and the stringent response. In fact, the stringent response signal, pppGpp, appears to be indispensible for transcriptional activation of curdlan biosynthesis.

Conclusions: This study identifies several mechanisms regulating the synthesis of curdlan, an EPS with numerous applications. These mechanisms are potential metabolic engineering targets for improving the industrial production of curdlan from Agrobacterium sp. ATCC 31749. Furthermore, many of the genes identified in this study are highly conserved across microbial genomes, and we propose that the molecular elements identified in this study may serve as universal regulators of microbial EPS synthesis.
\end{abstract}

Keywords: Agrobacterium, Curdlan, ATCC 31749, Exopolysaccharide, Exopolysaccharide regulation, EPS transcriptional regulation

\section{Background}

Microbial exopolysaccharides (EPS) are a class of natural products with stunning chemical diversities, which are comprised of a combination of constituent sugars, many different types of glycosidic linkages, stereochemistry, and additional chemical modifications on the sugars. In parallel to their chemical diversity, they display diverse physical properties that make them useful in many different applications. For their microbial producers, EPS

\footnotetext{
* Correspondence: rchen@chbe.gatech.edu

School of Chemical and Biomolecular Engineering, Georgia Institute of Technology, 311 Ferst Drive, Atlanta, GA 30332-0100, USA
}

serve important physiological functions, including cell adhesion, biofilm formation, symbiosis, protection from desiccation, and evasion of the human immune system [1]. Despite the chemical, physical, and physiological diversity they display, an outstanding common feature is that they are synthesized under adverse growth conditions. Often, their synthesis is triggered by a nutritional stress, and significant accumulation is only observed in the stationary phase [2]. As such, it is conceivable that there may be shared mechanisms by which microbes translate environmental cues into intracellular signals and subsequently activate the machinery for EPS

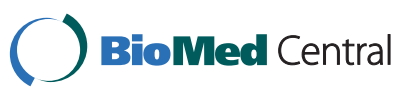


synthesis. Another intriguing fact is that EPS synthesis entails a significant input of cellular resources at a time when these resources are limited. There may be unifying mechanisms by which cells channel limited resources for EPS synthesis. As a first step to elucidate these possible conserved mechanisms, we apply transcriptome analysis to identify molecular components that may play roles in signal transduction of nutritional limitation, energy storage, and stress responses. We use curdlan synthesis in Agrobacterium sp. ATCC 31749 as our model system.

Agrobacterium sp. ATCC 31749 is a natural producer of curdlan EPS and is employed for large-scale curdlan production. Curdlan is used in a wide-range of applications including the production of superworkable concrete, as a gelling agent in the food industry, and as a main component of potential anti-tumor and anti-HIV treatments [3]. Despite the economic importance of curdlan, relatively little is known regarding the genetics and regulation of curdlan synthesis in ATCC 31749. Four genes are required for curdlan biosynthesis: $c r d A$, $c r d S$, $c r d C$, and $c r d R$ [4]. Curdlan synthesis is in response to nitrogen limitation, and two components of the nitrogen signaling cascade, $\mathrm{NtrB}$ and $\mathrm{NtrC}$, were reported to be essential for curdlan biosynthesis [3]. In other well-characterized microorganisms like E. coli, nitrogen-limited processes are transcriptionally regulated via the nitrogen signaling cascade, in which $\mathrm{NtrC}$ activates transcription via the $\sigma^{\mathrm{N}}$-containing form of RNA polymerase [5]. If this model holds true for curdlan synthesis, the sigma factor $\sigma^{\mathrm{N}}$ would play an important role in the transcriptional regulation of the curdlan operon. Our recent genome sequencing of ATCC 31749 [6] suggests the presence of a bacterial organelle, known as an acidocalcisome, in this strain, similar to the closely related Agrobacterium tumefaciens [7]. This is potentially relevant to curdlan synthesis as the organelle is specifically involved in the storage and metabolism of cellular polyphosphate (polyP) [8]. Kornberg and his coworkers suggested that polyP, linear polymers of tens or hundreds of orthophosphate residues linked by high-energy phosphoanhyride bonds, can substitute for ATP, among other functions, in a variety of cellular processes [9]. They later found that polyP levels are co-regulated with AlgR, a regulatory protein for the synthesis of alginate, an EPS synthesized by Pseudomonas aeruginosa [10]. Moreover, they found a link between polyP accumulation and nitrogen limitation [11]. The connection of EPS synthesis to polyP, however, is complicated by the fact that polyP may serve as not only a potential ATP substitute, but also as a regulator of cellular functions involved in stress response and cell survival, which may also be important for EPS synthesis [12]. The genome sequence also indicates the presence of numerous GGDEF-containing proteins associated with the synthesis and degradation of bis- $\left(3^{\prime}, 5^{\prime}\right)$-cyclic-dimeric-guanosine monophosphate (cdi-GMP), previously shown to play a role in regulating EPS synthesis [13], suggesting another potential conserved mechanism of regulation for EPS biosynthesis.

The understanding of EPS biosynthesis regulation is most advanced for alginate synthesis in Pseudomonas aeruginosa, owing to the importance of alginate in pathogenesis. The current model, as reviewed by Rehm and Valla, depicts a complex network that involves multiple environmental sensing and signal transduction elements, regulatory proteins both global (catabolite activator protein) and specific to alginate (AlgR), and a specific sigma factor (AlgU) [14]. We expect the regulatory circuits of curdlan biosynthesis will also be multifaceted, and thus, the application of systems biology tools will be most fruitful in identifying the factors involved in the process. In this work, we first use transcriptome profiling to identify genes that are differentially expressed during curdlan synthesis and subsequently carry out targeted gene knockouts to investigate their roles in the transcriptional regulation of curdlan production. We focus on the signal transduction of nitrogen limitation, the potential role of acidocalcisomes for energy provision, and the stringent response, as we believe these aspects, to some degree, are shared by other microbial EPS.

\section{Results}

\section{Transcriptome analysis of ATCC 31749}

Like many other EPS, curdlan biosynthesis is triggered by nutrient limitation, specifically nitrogen starvation [15]. To investigate the regulation of curdlan synthesis, a custom DNA microarray was designed using the draft genome sequence of ATCC 31749, and transcriptome analysis was conducted on samples taken at 22 hours, during the exponential growth phase without curdlan production, and at approximately 70 hours after nitrogen depletion, during curdlan production (Figure 1). The microarray data was deposited in Gene Expression Omnibus (GEO accession number: GSE32576). A total of 2,456 genes had more than a 2 -fold change in gene expression level during curdlan production (Table 1), of which 985 were up-regulated and 1,471 were downregulated. Notably, the curdlan biosynthesis operon ( $c r d A S C)$ was up-regulated by up to 100-fold (Table 2), providing the first experimental evidence of curdlan transcriptional regulation. Genes with significant expression changes were grouped according to function (Table 1 ). A majority of the up-regulated genes (55\%) code for hypothetical proteins of unknown function, which contributes to the difficulty in elucidating the regulatory mechanism of curdlan biosynthesis. Besides hypothetical proteins, genes associated with transporters, metabolism and cofactor synthesis, and regulation also experienced 


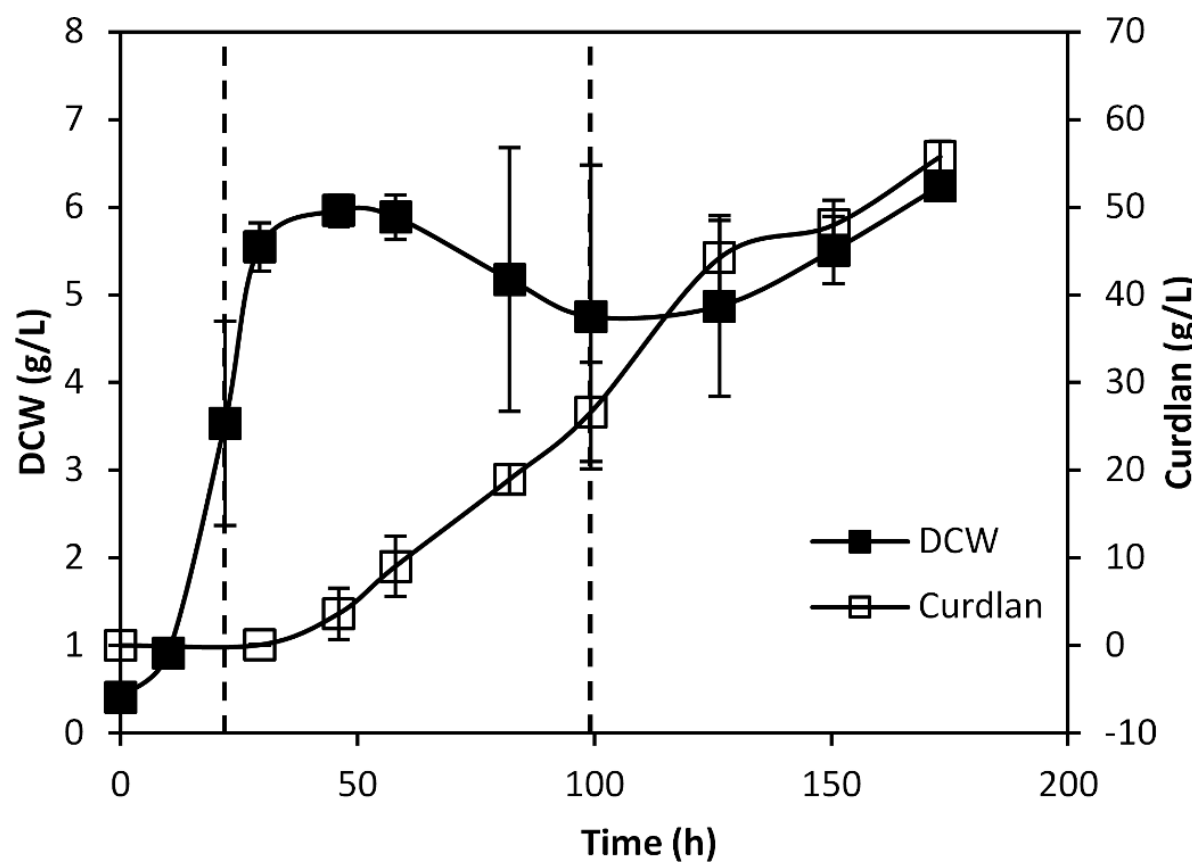

Figure 1 Dry cell weight (DCW) and curdlan concentration during cultivation of ATCC 31749. Vertical lines indicate sampling times for gene expression analysis. Error bars represent the standard deviation of 3 biological replicates.

significant changes (Table 1), suggesting extensive responses to the condition that triggers curdlan production.

Many mechanisms may regulate or influence curdlan biosynthesis, including the nitrogen signaling cascade, nucleotide-based second messengers such as c-di-GMP,

Table 1 Functional distribution of genes up and downregulated during curdlan biosynthesis

\begin{tabular}{lcccc}
\hline Functional Category & \multicolumn{2}{c}{ Up-regulated } & \multicolumn{2}{c}{ Down-regulated } \\
\cline { 2 - 6 } & $\begin{array}{c}\text { \# of } \\
\text { genes }\end{array}$ & $\%$ & $\begin{array}{c}\text { \# of } \\
\text { genes }\end{array}$ & $\%$ \\
\hline Cell replication and division & 43 & 4.4 & 82 & 5.6 \\
\hline $\begin{array}{l}\text { Membrane and lipid } \\
\text { metabolism }\end{array}$ & 18 & 1.8 & 69 & 4.7 \\
\hline $\begin{array}{l}\text { Protein synthesis and } \\
\text { degradation }\end{array}$ & 31 & 3.1 & 104 & 7.1 \\
\hline RNA-associated proteins & 4 & 0.4 & 74 & 5.0 \\
\hline Transporters & 86 & 8.7 & 210 & 14.3 \\
\hline $\begin{array}{l}\text { Metabolism and cofactor } \\
\text { synthesis }\end{array}$ & 111 & 11.3 & 381 & 25.9 \\
\hline Regulation & 105 & 10.7 & 101 & 6.9 \\
\hline Stress response/cell protection & 27 & 2.7 & 26 & 1.8 \\
\hline EPS synthesis and degradation & 8 & 0.8 & 24 & 1.6 \\
\hline Hypothetical proteins & 546 & 55.4 & 395 & 26.9 \\
\hline $\begin{array}{l}\text { Phage/invasion/virulence } \\
\text { proteins }\end{array}$ & 6 & 0.6 & 5 & 0.3 \\
\hline Total & $\mathbf{9 8 5}$ & $\mathbf{1 0 0 . 0}$ & $\mathbf{1 4 7 1}$ & $\mathbf{1 0 0 . 0}$ \\
\hline
\end{tabular}

energy storage in acidocalcisomes, and the stringent response (Figure 2). Differentially expressed genes associated with these potential regulatory mechanisms were selected for further experimental analysis (Table 2).

\section{Transcriptional regulation of curdlan production under nitrogen-limitation}

In bacteria and euryarchaeota, most nitrogen regulatory mechanisms are centrally controlled by a conserved and ancient set of nitrogen sensor proteins called PII [5] (Figure 2), and the signal is transmitted by a two component signal transduction mechanism involving $\mathrm{NtrB}$ and $\mathrm{NtrC}$. In the cascade, $\mathrm{NtrC}$ is phosphorylated by $\mathrm{NtrB}$ under nitrogen-deplete conditions. In turn, phosphorylated NtrC binds to the RpoN sigma factor to initiate transcription [5]. Previously, NtrB and NtrC, were shown to be necessary for curdlan biosynthesis in ATCC 31749 [3]. The genome of this strain encodes a full complement of genes for nitrogen sensing and signal transduction, but curiously, the $n \operatorname{tr} B C$ operon also contains nifR, whose function is unknown but appears as the first gene of the $n \operatorname{tr} B C$ operon in nitrogen-fixing $\alpha$ proteobacteria. Thus, NifR appears to be associated with nitrogen fixation, yet, the draft genome of ATCC 31749 lacks a nitrogenase, rendering this strain incapable of nitrogen fixation. The transcriptome analysis showed that expression of nifR increased 2-fold during curdlan synthesis (Table 2). To determine whether nifR encodes a protein that is relevant for curdlan biosynthesis, a 
Table 2 Gene expression changes during curdlan production for select genes with potential influence on curdlan biosynthesis

\begin{tabular}{|c|c|c|c|c|}
\hline Gene ID & Fold Change & P-value & Gene Symbol & Description \\
\hline \multicolumn{5}{|c|}{ Curdlan biosynthesis } \\
\hline AGRO_1847 & 99.0 & $7.8 \mathrm{E}-4$ & $\operatorname{crd} A$ & hypothetical protein Atu3057 \\
\hline AGRO_1848 & 94.8 & $1.1 \mathrm{E}-3$ & crds & $\beta 1,3$-glucan synthase catalytic subunit \\
\hline AGRO_1849 & 65.8 & $9.1 \mathrm{E}-5$ & $\operatorname{crdC}$ & hypothetical protein Atu3055 \\
\hline$\overline{\text { AGRO_1729 }}$ & 47.5 & $4.8 \mathrm{E}-5$ & - & ECF family RNA polymerase sigma factor \\
\hline \multicolumn{5}{|c|}{ Nitrogen signaling cascade } \\
\hline AGRO_1033 & 5.2 & $1.4 \mathrm{E}-2$ & glnK & nitrogen regulatory protein PII \\
\hline AGRO_4555 & 2.0 & $1.8 \mathrm{E}-2$ & nifR & nitrogen regulation protein \\
\hline AGRO_0407 & 1.9 & $8.3 \mathrm{E}-3$ & rpoN & RNA polymerase factor sigma-54 \\
\hline AGRO_4554 & -1.0 & $8.1 \mathrm{E}-1$ & $n \operatorname{tr} B$ & two component sensor kinase \\
\hline AGRO_4553 & -2.0 & $9.4 \mathrm{E}-2$ & $n$ trC & two component response regulator \\
\hline AGRO_0420 & -5.8 & $1.0 \mathrm{E}-3$ & $g \ln D$ & PIl uridylyl-transferase \\
\hline \multicolumn{5}{|c|}{ c-di-GMP biosynthesis } \\
\hline AGRO_3967 & 5.7 & $3.2 \mathrm{E}-3$ & - & GGDEF domain protein \\
\hline AGRO_0636 & 2.3 & $5.7 \mathrm{E}-2$ & - & GGDEF domain protein \\
\hline AGRO_0033 & 2.2 & $4.2 \mathrm{E}-2$ & - & GGDEF domain protein \\
\hline \multicolumn{5}{|c|}{ Acidocalcisome-associated } \\
\hline AGRO_5348 & 2.4 & $5.1 \mathrm{E}-4$ & chaA & $\mathrm{Ca}^{2+} / \mathrm{H}^{+}$antiporter \\
\hline$\overline{\text { AGRO_2774 }}$ & 1.9 & $3.3 \mathrm{E}-3$ & $p p \times 1$ & exopolyphosphatase \\
\hline AGRO_2974 & 1.8 & 3.0E-1 & aqpZ & aquaporin Z \\
\hline AGRO_2553 & 1.1 & $4.4 \mathrm{E}-1$ & ppk & polyphosphate kinase \\
\hline AGRO_1846 & 1.1 & $6.7 \mathrm{E}-1$ & - & $\mathrm{Na}^{+} / \mathrm{H}^{+}$antiporter \\
\hline AGRO_0927 & -2.2 & $6.8 \mathrm{E}-4$ & ppa & Inorganic pyrophosphatase \\
\hline GRO_2552 & -3.6 & $2.5 \mathrm{E}-3$ & $p p \times 2$ & exopolyphosphatase \\
\hline$\overline{A G R O \_2518}$ & -6.9 & $4.5 \mathrm{E}-3$ & $\operatorname{rrp} P$ & membrane-bound proton-translocating pyrophosphatase \\
\hline \multicolumn{5}{|c|}{ Stringent response } \\
\hline$\overline{A G R O \_1497}$ & 3.9 & 1.6E-3 & relA/spoT & GTP pyrophosphohydrolase/synthetase \\
\hline
\end{tabular}

knockout mutant was generated. As shown in Figure $3 \mathrm{~A}$, the nifR mutant was able to synthesize curdlan, yet the production of curdlan was reduced to $30 \%$ of the wild type production. While we cannot rule out polar effects from deletion of nifR on $n t r B C$ transcription, these results confirm the link between curdlan biosynthesis and nitrogen-limited regulation.

To determine if the classical mechanism of the nitrogen signaling cascade regulates transcription of the curdlan synthesis operon, the sigma factor $r p o N$ was targeted for gene knockout. Surprisingly, the rpoN mutant not only synthesized curdlan (Figure 3A), but it produced over 30\% more curdlan than the wild type. This result indicates that the regulation of curdlan biosynthesis is RpoN-independent, implicating an alternative sigma factor for transcriptional activation of the curdlan synthesis operon. Furthermore, a 30\% increase in curdlan production for $\Delta r p o N$ suggests that sigma factor competition may occur, whereby deletion of RpoN reduces sigma factor competition for RNA polymerase and allows for increased curdlan biosynthesis through the yet unknown alternative sigma factor [16]. One plausible candidate sigma factor is AGRO_1729, an extracytoplasmic function (ECF) sigma factor, which displayed a 47-fold increase in gene expression during curdlan production (Table 2). As shown in Figure 3A, the AGRO_1729 knockout mutant produced curdlan at levels similar to the wild-type, suggesting its minimal involvement in regulating curdlan synthesis. While the sigma factor responsible for the transcriptional regulation remains elusive, the data presented here confirmed the role of $n t r B C$ operon in the signal transduction but the mechanism involving the $n t r B C$ operon seems to be unique for curdlan synthesis in ATCC 31749.

\section{Curdlan synthesis is influenced by the nucleotide second messenger, c-di-GMP}

Exopolysaccharides, such as cellulose, alginate, and Pel polysaccharide, are regulated by the nucleotide second 


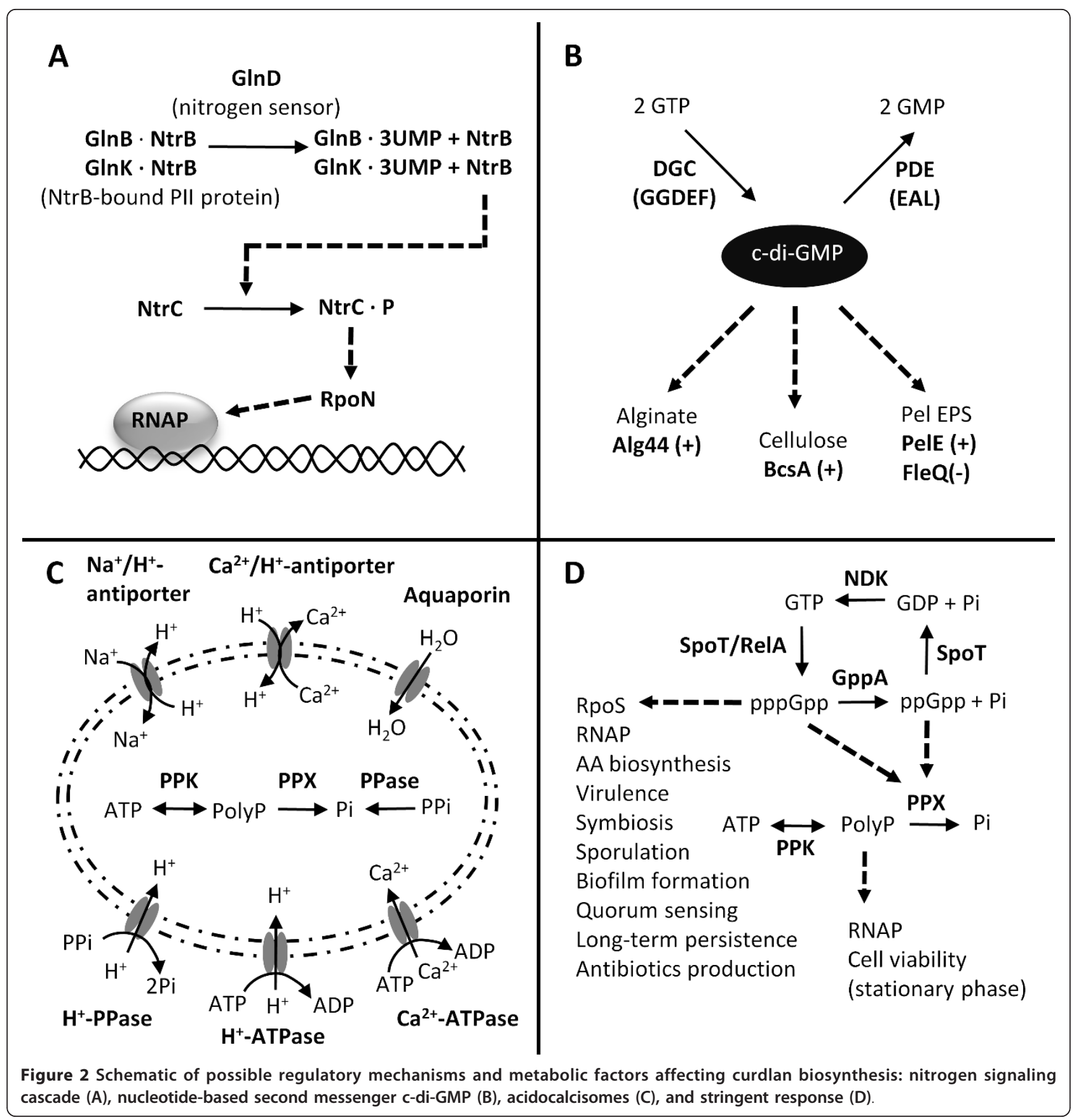

messenger, c-di-GMP. C-di-GMP acts as an allosteric regulator of cellulose and alginate biosynthesis, and it serves as both an allosteric and transcriptional regulator of Pel EPS in Pseudomonas aeruginosa [13]. C-di-GMP is synthesized by diguanylate cyclases which contain a conserved GGDEF motif as the catalytic active site. The genome sequence of ATCC 31749 contains 31 predicted genes coding for GGDEF domain proteins which may control c-di-GMP levels and potentially regulate curdlan biosynthesis. Only 3 GGDEF domain proteins had more than a 2-fold up-regulation in gene expression under nitrogen limitation (Table 2); the corresponding genes were targeted for deletion.

The AGRO_3967 mutant showed a 57\% decrease in curdlan production (Figure 3A), suggesting that c-diGMP may regulate curdlan biosynthesis. The other GGDEF domain mutants $(\Delta 0033$ and $\Delta 0636)$, however, produced curdlan at levels similar to that of the wildtype. These results correlate with the measured gene expression levels. AGRO_3957 displayed the largest 


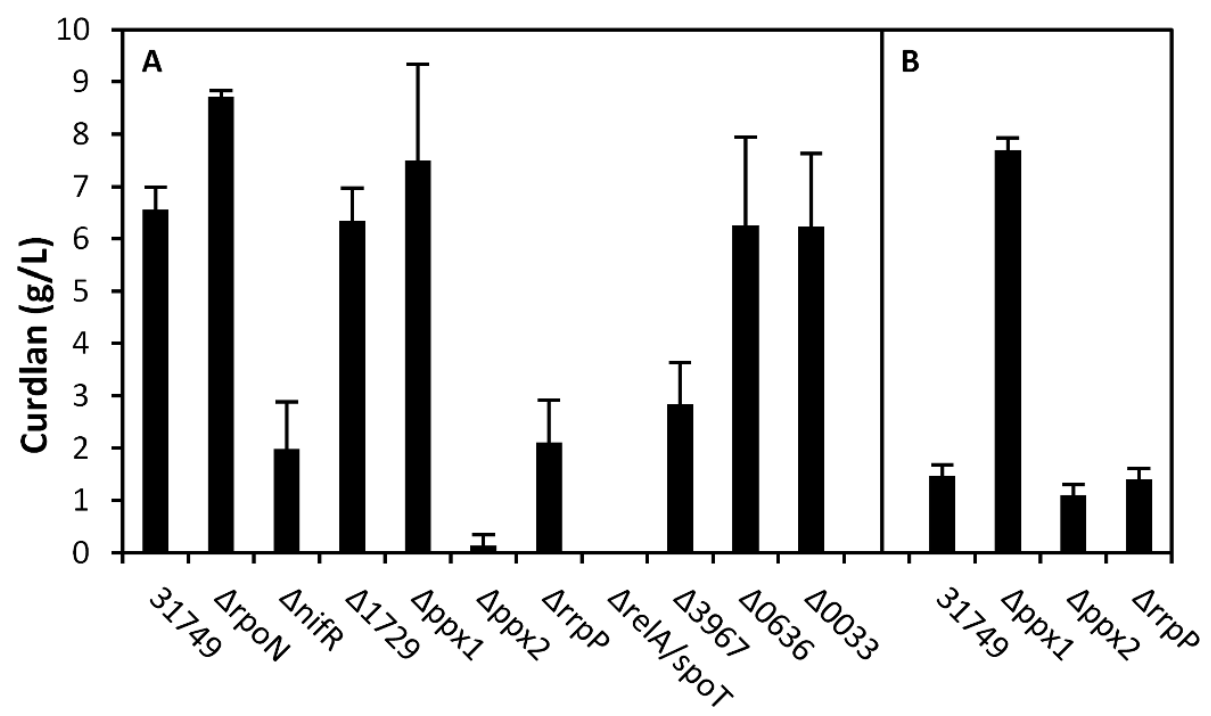

Figure 3 Curdlan biosynthesis in ATCC 31749 and gene knockout mutants after 24 hours of cultivation in nitrogen-free media using stationary phase (A) and late exponential phase (B) cells. Error bars represent the standard deviation of 3 biological replicates.

increase in gene expression (5.7-fold), and its associated mutant had impaired curdlan production. On the other hand, AGRO_0033 and AGRO_0636 not only had less of an increase in gene expression $(<2.4$-fold), but the statistical significance of increased gene expression was low for these GGDEF domain genes (p-values > 0.042). Accordingly, curdlan production was unaffected in the corresponding AGRO_0033 and AGRO_0636 knockout mutants. Overall, these results suggest c-di-GMP levels in ATCC 31749 affect curdlan biosynthesis, and more specifically, c-di-GMP synthesis via AGRO_3967 is important for high curdlan production.

\section{Acidocalcisomes and PolyP metabolism influence curdlan synthesis}

Curdlan biosynthesis is an energy-intensive process, requiring two high-energy molecules for each nascent glycosidic bond. In fact, energy availability was shown to be a limiting factor of curdlan biosynthesis in ATCC 31749 [17]. One potential source of energy is polyP, a phosphate polymer containing many high-energy bonds. In Agrobacterium tumefaciens, a close relative of ATCC 31749 , polyP accumulates in intracellular organelles known as acidocalcisomes [7]. The ATCC 31749 genome contains many genes associated with acidocalcisomes, including those listed in Table 2. The influence of acidocalcisomes and polyP on curdlan biosynthesis was investigated.

The accumulation of polyP in acidocalcisomes is enhanced under stationary phase or stress conditions [18]. In E. coli, for example, the level of polyP is low in the exponential phase and increases up to 1000-fold upon amino acid starvation [19]. Nitrogen limitation also elicits a high level of polyP accumulation [11]. Both nitrogen depletion and the stationary phase are also required for optimal curdlan production, providing the first clue that polyP may be related to curdlan production. Cells harvested from stationary phase produced 6.5 $\mathrm{g} / \mathrm{L}$ of curdlan after 24 hours of cultivation under nitrogen deplete conditions, whereas cells harvested from exponential phase only produced $1.5 \mathrm{~g} / \mathrm{L}$ under the same conditions (Figure 3 ). The level of poly $\mathrm{P}$ is influenced by the activity of exopolyphosphatase (PPX), an enzyme that hydrolyzes a phosphate molecule from the polymer chain of polyP. As shown in Figure 3B, knockout of $p p x 1$ (encoding a putative exopolyphosphatase) led to significant increase in curdlan synthesis compared to wild type cultures for cells harvested from the exponential phase. Eliminating exopolyphosphatase activity allows polyP to accumulate during the exponential phase, subsequently leading to curdlan production at the high levels traditionally observed only with stationary phase cultures. In contrast, knockout of $p p x 1$ has no effect on curdlan synthesis for stationary phase cells (Figure 3A), consistent with the expectation that exopolyphosphatase activity is already low in the stationary phase due to the high level of (p)ppGpp, an inhibitor to PPX which typically accumulates in the stationary phase [19]. These results suggest that polyP may serve as an important energy source for curdlan biosynthesis in ATCC 31749. It is important to note that the genome encodes two PPXs but experimental evidence showed PPX2 behaves quite differently from PPX1 and does not likely use polyP as substrate.

Previous studies showed strong correlation of $\mathrm{pH}$ and curdlan synthesis, with pH 5.5 being optimal for curdlan 
synthesis [20], significantly lower than its optimum for growth ( $\mathrm{pH} 7.0)$. In addition to the phosphate and energy storage functions associated with polyP accumulation, acidocalcisomes could also influence curdlan synthesis through $\mathrm{pH}$ regulation, as the organelle is also known to play important role in maintenance of intracellular $\mathrm{pH}$ [8]. The putative acidocalcisome gene with a role in regulating $\mathrm{pH}$ is $\operatorname{rrpP}$, a membrane-bound proton-translocating pyrophosphatase, which showed nearly a 7-fold drop in gene expression under curdlan-producing conditions (Table 2). Additionally, gene knockout of $\operatorname{rrpP}$ led to nearly a $70 \%$ decrease in curdlan production compared to the wild type (Figure $3 \mathrm{~A}$ ), indicating that proton translocation between the cytosol and acidocalcisome, mediated by RrpP, is important for optimum curdlan synthesis.

Taken together, the experimental data from this study provides the first evidence to link the acidocalcisome with curdlan synthesis through both high energy provision and $\mathrm{pH}$ regulation.

\section{The stringent response regulates transcription of the curdlan synthesis operon}

In addition to its potential as a high energy storage compound, polyP accumulation is an essential element of the stringent response (Figure 2). The stringent response was first studied in E. coli as a response to amino acid starvation, but it has since been linked with other stress conditions including nitrogen limitation $[11,21]$. During the stringent response, guanosine pentaphosphate (pppGpp) and guanosine tetraphosphate (ppGpp), collectively known as (p)ppGpp, accumulate within the cell. Besides acting as allosteric inhibitors of exopolyphosphatase, (p)ppGpp can directly influence gene transcription and translation [21].

The previous section on polyP metabolism describes two predicted ppx genes in ATCC 31749, yet experimental evidence suggests only one gene product ( $p p x 1)$ has exopolyphosphatase activity. The other predicted exopolyphosphatase gene ( $p p x 2)$ likely has phosphatase activity, yet it may utilize pppGpp as substrate rather than polyP. From its amino acid sequence, $p p x 2$ has two predicted catalytic domains, one for exopolyphosphatase (PPX) activity and one for guanosine pentaphosphate phosphohydrolase (GppA) activity. As additional evidence that PPX2 does not exhibit exopolyphosphatase activity, the level of polyP during growth of $\Delta p p x 2$ was not enhanced compared to polyP levels in the wild type (Figure 4). If PPX2 functions primarily as a GppA, $p p x 2$ deletion would yield high levels of pppGpp and low levels of ppGpp. Stationary phase cultures of $\Delta p p x 2$ show nearly a $98 \%$ reduction in curdlan biosynthesis (Figure $3 \mathrm{~A}$ ), suggesting that ppGpp may be the active form of (p)ppGpp for the stringent response in ATCC 31749. Since (p)
ppGpp only accumulates under stationary phase conditions, knockout of $p p x 2$ is not expected to influence curdlan production in exponential phase cultures. In agreement with this, exponential phase cultures of $\Delta p p x 2$ produced curdlan at levels similar to the wild type (Figure $3 \mathrm{~B})$. Interestingly, the $p p x 2$ mutant also showed reduced cell viability under nitrogen limitation (Figure 5). This behavior is similar to that observed in both polyP-and ppGpp-deficient E. coli cells which are not able to survive under stationary phase conditions [22,23]. The reduced viability of $\Delta p p \times 2$ during stationary phase suggests that the ppGpp form of (p)ppGpp is important for activating stress responses in ATCC 31749.

The stringent response factor (p)ppGpp is synthesized in E. coli by two different enzymes: RelA, a (p)ppGpp synthetase, and SpoT, a bifunctional (p)ppGpp synthetase/ ppGpp 3'-phosphohydrolase [16]. Using E. coli RelA and SpoT sequences (Accession numbers: NP_417264 and ACB04699) as query, BLASTP identified AGRO_1497 as the only RelA and SpoT homolog in ATCC 31749, having $31 \%$ and $37 \%$ identity with the E. coli sequences respectively. It appears that this single relA/spoT homolog controls both (p)ppGpp synthesis and degradation. Deletion of relA/spoT completely eliminates curdlan synthesis in ATCC 31749 under nitrogen-limited conditions (Figure $3 \mathrm{~A})$. A reduction in curdlan biosynthesis may be explained by a decreased level of polyP due to elimination of the PPX inhibitor, (p)ppGpp. Yet, curdlan production is not simply reduced in the relA/spoT mutant; it is completely eliminated, suggesting the effect of (p)ppGpp extends beyond its inhibition of PPX. Based on the results from both the ppx2 (putative GppA) and relA/spoT mutants, the stringent response signal, (p)ppGpp, is clearly essential for curdlan biosynthesis.

(p)ppGpp is a global regulator of gene expression in $E$. coli and other bacteria [16]. Thus, to determine if (p) ppGpp is involved in the transcriptional regulation of the curdlan synthesis operon (crdASC), gene expression levels of curdlan synthase $(c r d S)$ in the $r e l A / s p o T$ and $p p x 2$ mutant strains were compared to wild type. Under nitrogen limitation, $\Delta r e l A / s p o T$ cultures exhibited $c r d S$ expression levels that were up to 57 -fold lower than in wild type cultures. This demonstrates that (p)ppGpp is involved in the activation of transcription of curdlan synthase genes. As discussed above, knockout of the putative GppA, $p p x 2$, led to impaired curdlan production; however, $c r d S$ expression levels were only reduced up to 2 -fold in $\Delta p p \times 2$ compared to wild type. This may indicate that the two forms of (p)ppGpp, pppGpp and ppGpp, have differential mechanisms of regulating curdlan synthesis.

\section{Discussion and conclusions}

Microbial EPS are often synthesized in response to a nutritional stress, with nitrogen limitation being a 


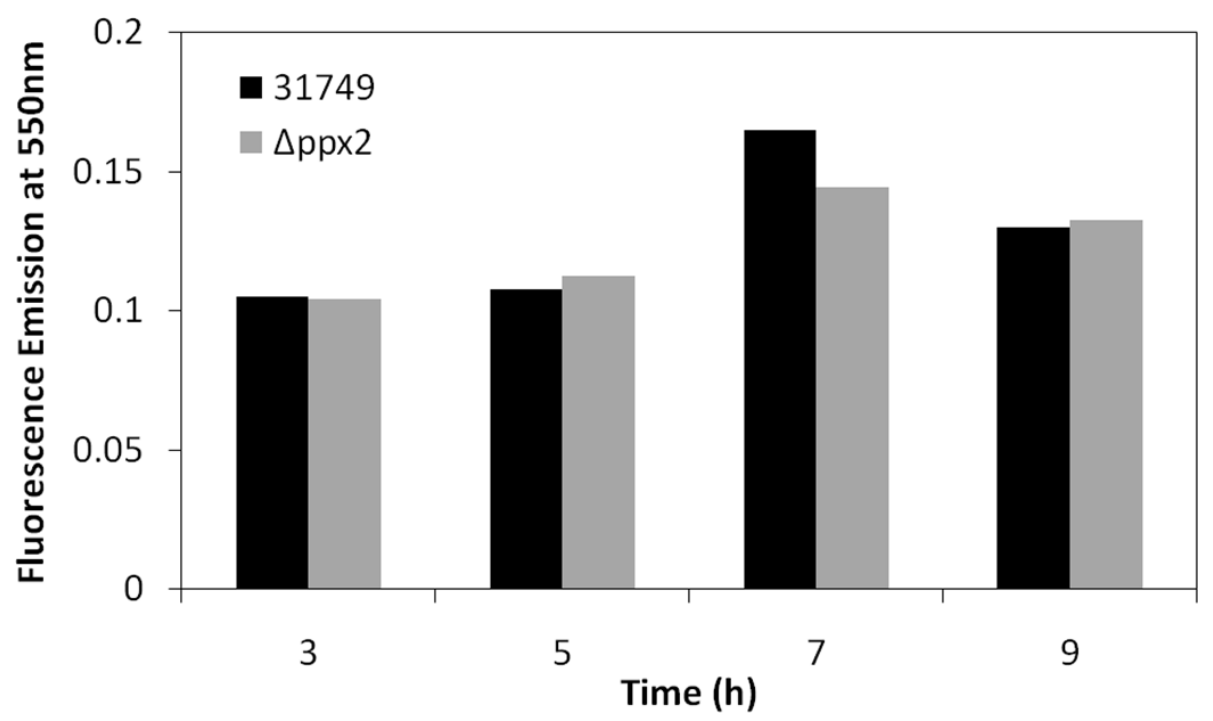

Figure 4 Relative polyP levels in wild type (31749) and $\Delta p p x 2$ during exponential growth.

common environmental cue. As EPS synthesis entails a significant investment in terms of cellular energy and other resources under a condition when resources and energy are scarce, extensive re-organization of metabolic activity is expected and sophisticated regulatory mechanisms are needed to respond to environmental triggers. The common trigger of nutrient limitation for their synthesis and their association with adverse growth conditions suggest some unifying scheme of EPS regulation. Yet, our understanding is very limited about how their biosynthesis is regulated at the molecular level. In this study, we probe the regulation mechanisms of curdlan synthesis with a focus on those potentially conserved mechanisms. The transcriptome profiling along with genetic, biochemical, and physiological analysis reveals a multifaceted network of regulation for curdlan biosynthesis, including RpoN-independent nitrogen regulation, c-di-GMP, acidocalcisomes, polyP metabolism, and the stringent response.

First, the transcriptome analysis showed that upon nitrogen depletion, the curdlan synthesis operon was up-regulated by up to 100-fold, thus transcriptional regulation plays a prominent role in its biosynthesis. As curdlan synthesis is activated in response to nitrogen depletion, the involvement of the nitrogen signaling cascade is expected. The signal transduction components,

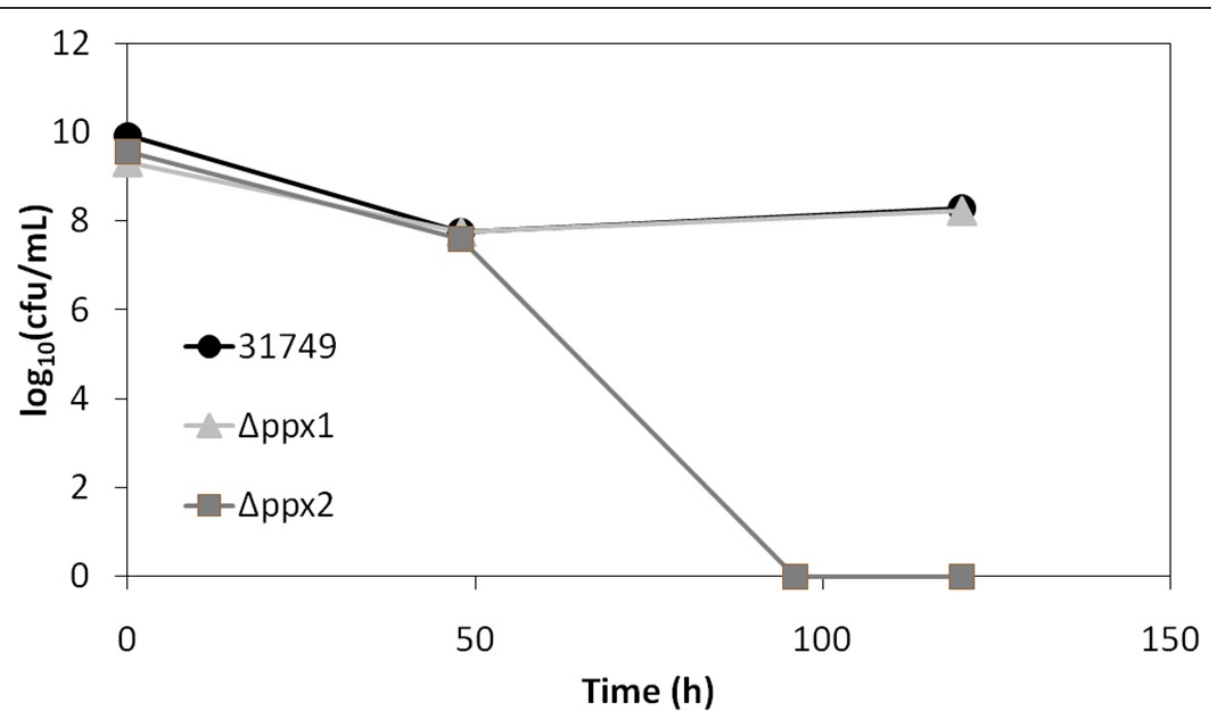

Figure 5 Cell viability of wild type (31749), $\Delta p p x 1$, and $\Delta p p \times 2$ during curdlan production measured by colony forming units (cfu). 
$\mathrm{NtrB}$ and NtrC, were previously identified as essential components for curdlan synthesis [3], and this was confirmed in this study. Additionally, this study uncovered some unexpected details of how nitrogen signaling affects curdlan synthesis. The operon structure for $n \operatorname{tr} B C$, having $n i f R$ as the first gene, is unusual for a non-nitrogen fixer, and nifR deletion was found to reduce curdlan production. We also show that the RpoN sigma factor is not involved, implicating an alternative sigma factor in the regulation. This is a significant departure from the archetype nitrogen regulation, in which RpoN is required for transcription of the regulated genes and NtrC serves as the activator. RpoNindependent transcriptional regulation has been shown to operate extensively in only one other microorganism, Rhodobacter capsulatus, a photosynthetic bacterium and a nitrogen fixer [24]. Interestingly, $R$. capsulatus also contains a nifR-like gene (nifR3) in the same operon as $n \operatorname{tr} B C$ [25], suggesting a connection between the $n \operatorname{tr} B C$ operon structure and its RpoN independence. Future studies to identify the elusive sigma factor responsible for the regulation holds the key to understand this new mode of nitrogen signaling.

The genome sequence of ATCC 31749 suggests the presence of aciocalcisomes. This study provides the first evidence that metabolism within the organelle influences curdlan biosynthesis through energy storage in the form of polyP and maintenance of intracellular $\mathrm{pH}$. The reduced curdlan produced by $\Delta r r p P$ suggests that the membrane-bound proton-translocating pyrophosphatase (RrpP) plays an essential role in acidocalcisomemediated intracellular $\mathrm{pH}$ regulation. By eliminating polyP degradation via $p p x 1$ deletion, high curdlan synthesis could be triggered in exponential phase cells, clearly establishing the role of polyP in curdlan biosynthesis. The contribution of polyP as an energy source in curdlan biosynthesis, however, is complicated by the regulatory role of polyP in the stringent response.

Despite the fact that many EPS are produced under nutritionally stressed conditions which likely trigger a stringent response, bacterial EPS synthesis have rarely been studied in the context of the stringent response. In this study, we show that curdlan synthesis is abolished in cells lacking the gene responsible for synthesis of the stringent response signal (p)ppGpp. This indicates that curdlan synthesis requires the stringent response. Further analysis by qRT-PCR showed that the curdan synthesis operon was down-regulated by 57 -fold in the relA/spoT deletion mutant. Thus without the stringent response, up-regulation of the curdlan operon could not be elicited. This is a significant finding as it convincingly establishes requirement of the stringent response for the transcriptional up-regulation of an EPS operon. Only one previous report described a relationship between the stringent response and EPS synthesis [26]. However, in this report, the Sinorhizobium meliloti mutant incapable of synthesizing the effector molecule of stringent response by knocking out the only $\mathrm{relA} / \mathrm{spoT}$ gene (the same approach as used in this study), produced more succinoglycan, an EPS, and the succinoglycan operon was significantly up-regulated in the mutant. Thus, in this case, ppGpp negatively regulates succinoglycan synthesis, which is significantly different from the complete dependence of curdlan synthesis on the presence of the effector molecule, (p)ppGpp. Hence, our data suggest a novel type of EPS regulation as a direct effect of the stringent response, which has not been reported. The molecular details of activation, however, await further elucidation. As reviewed by Srivatsan and Wang, two models of activation were proposed for (p)ppGpp [21]. In direct activation, the effector molecule, (p) ppGpp, increases transcription from cognate promoters by acting on RNA polymerase, whereas indirect activation involves an alternative sigma factor. Much of the understanding of the stringent response, however, is based on the studies with $E$. coli, in which the stationary phase sigma factor, RpoS, plays a central role in stringent response [23]. Unfortunately, the E. coli model is unlikely to be applicable here as the ATCC 31749 genome does not encode a corresponding stationary phase sigma factor. Additionally, the ATCC 31749 genome contains only one relA/spoT homolog and two predicted exopolyphosphatases, signifying a significant departure from the $E$. coli system.

Additional data were obtained, which demonstrated the influence of the nucleotide second messenger, c-diGMP, on curdlan synthesis. C-di-GMP is synthesized by diguanylate cyclases which contain a conserved GGDEF motif as the catalytic active site [13]. The genome sequence of ATCC 31749 contains 31 predicted genes coding for GGDEF domain proteins, of which three GGDEF domain proteins had more than a 2 -fold upregulation in gene expression under nitrogen limitation (Table 2). Deletion of AGRO_3967, encoding an upregulated GGDEF domain protein, yielded a $57 \%$ decrease in curdlan production (Figure 3A), suggesting that $\mathrm{c}$-di-GMP may regulate curdlan biosynthesis, thus adding another factor to the already complicated regulatory network.

The use of systems biology tools in this study identified many components important to the transcriptional regulation of curdlan synthesis, including the $n \operatorname{tr} B C$ operon in nitrogen signal transduction, polyP, (p)ppGpp, and c-di-GMP. Subsequent experiments with gene knockout mutants and qRT-PCR revealed some aspects that are unique to curdlan synthesis and the producer microorganism. These include RpoN-independent activation of the curdlan operon and the connection of 
curdlan synthesis to the metabolic activity of a bacterial organelle. We also present convincing evidence that curdlan biosynthesis is involved in the stringent response. While these three aspects appear to be unique to curdlan, the components identified, NtrBC, polyP, (p) ppGpp, and c-di-GMP, are present in most microorganisms that synthesize EPS. Thus, it is reasonable to expect some common features to emerge. Indeed, Kornberg suggested that polyP may have served as an ATPalternative energy source [9], and subsequent work from his lab confirmed the role of polyP in alginate synthesis in $P$. aeruginosa [10], and in a separate work, demonstrated a connection between polyP accumulation, nitrogen limitation, and the stringent response [11]. Additionally, the stringent response in $P$. aeruginosa was recently shown to regulate quorum sensing [27], which affects synthesis of the EPS alginate [28], providing another link between the stringent response and EPS biosynthesis. Thus, polyP, a ubiquitous molecule accumulated in response to nitrogen limitation and in the stationary phase, plays a central role in the regulation of EPS synthesis and may additionally serve as an energy source. Our findings from this study further support this notion. In reference to the alginate model as reviewed by Rehm and Valla, a sigma factor (AlgU) and regulatory protein $(\mathrm{AlgR})$, are central to integrate the molecular components influencing alginate biosynthesis [14]. Data presented here indicate that an unidentified sigma factor is involved in the regulation, and previous studies implicate the presence of a regulatory protein (CrdR) required for curdlan synthesis [3]. Further studies with CrdR and identification of the yet unknown sigma factor involved in the transcriptional regulation will be important to integrate the information uncovered in this work. While we believe each regulatory network may differ in some specific details, NtrC, polyP metabolism, and stringent response are aspects that may provide a basis for unified mechanisms of EPS regulation.

\section{Methods}

\section{Bacterial strains and plasmids}

The bacterial strains and plasmids used in this study are listed in Table 3. For gene knockout, an internal fragment of the target gene (200-500 bp) was cloned and inserted into the SacI and HindIII sites of the suicide vector, pUCP30T, following the method outlined in [29]. The primers used for cloning the internal fragments are listed in Table 4. The knockout plasmids were transformed via electroporation using the BioRad micropulser Agr program. Knockout mutants were selected by screening on LB-agar plates containing 50 $\mu \mathrm{g} / \mathrm{mL}$ of gentamicin. Successful gene KO was confirmed with PCR using primers that span the junction between the chromosome and inserted $\mathrm{KO}$ plasmid (Table 4).

\section{Fermentation for curdlan synthesis}

ATCC 31749 colonies were inoculated into test tubes containing $4 \mathrm{~mL}$ of $\mathrm{LB}$ media and grown overnight at $30^{\circ} \mathrm{C}$ with $250 \mathrm{rpm}$. The inoculum was diluted $1500 \mathrm{x}$ in a 500 $\mathrm{mL}$ flask of $150 \mathrm{~mL} \mathrm{LB}$ media. After 17 hours of growth, the cells were concentrated by centrifugation $(3000 \times \mathrm{g}, 10$ min, $4^{\circ} \mathrm{C}$ ) to a volume of $30 \mathrm{~mL}$. The concentrated culture was added to $450 \mathrm{~mL}$ of minimal fermentation media [30] with $1.4 \mathrm{~g} / \mathrm{L}$ of $\mathrm{KH}_{2} \mathrm{PO}_{4}$ in an Infors Multifors fermenter system with four parallel $500 \mathrm{~mL}$ fermentation vessels. $\mathrm{NaOH}$ and $\mathrm{HCl}(1 \mathrm{M})$ solutions were used for $\mathrm{pH}$ control. Agitation was set at $600 \mathrm{rpm}$ with $1 \mathrm{vvm}$ for the air flowrate, and the $\mathrm{pH}$ was controlled at $\mathrm{pH} 7$ for growth. The ammonium level was measured using the Berthelot reaction as described by [31]. After nitrogen exhaustion, 10 $\mathrm{mL}$ samples were taken every 24 hours for 5 to 7 days. Curdlan and dry cell weight measurements were performed as described by [30].

\section{RNA isolation and microarray processing}

The RNA was immediately stabilized using Qiagen's RNAprotect Bacteria Reagent and stored at $-80^{\circ} \mathrm{C}$. RNA was isolated using Qiagen's RNeasy mini kit with oncolumn DNase digestion (15 mg/mL lysozyme, $15 \mathrm{~min}$ for lysis). For samples with high levels of curdlan, the volumes of lysozyme solution, RLT buffer, and ethanol were doubled, and a centrifugation step $(5,000 \times \mathrm{g}$ for 5 min at $10^{\circ} \mathrm{C}$ ) was added after the addition of RLT buffer. Contaminating genomic DNA was removed using Ambion's TURBO DNA-free kit. RNA quality was determined using Agilent's 2100 bioanalyzer with the RNA 6000 Nano kit. RNA concentration was calculated from $\mathrm{OD}_{260}$, measured using the SpectraMax M5 microplate reader with Corning's UV-transparent 96-well microplates. Purified RNA samples were stored at $-80^{\circ} \mathrm{C}$ until analysis.

The subsequent steps described in this paragraph were performed by Gene Logic, Inc. The RNA was converted to cDNA and labeled with either $\mathrm{Cy}-3$ or $\mathrm{Cy}-5$ using the Fairplay III Microarray Labeling kit from Agilent. The microarray is an $8 \times 15 \mathrm{k}$ custom DNA microarray from Agilent with probes designed using Agilent's eArray program. The eArray program designed probes for 5,580 of the 5,585 predicted genes in the ATCC 31749 genome with two technical replicates for each gene. For each condition, there are 4 biological replicates with dye swap. A microarray scanner model G2505B from Agilent Technologies was employed for microarray imaging, 
Table 3 Bacterial strains and plasmids used in this study

\begin{tabular}{|c|c|c|}
\hline Strain/Plasmid & Description & Source \\
\hline JM109 & E. coli K12 strain used for cloning & Promega \\
\hline ATCC 31749 & Curdlan-producing Agrobacterium sp. (wild-type) & ATCC \\
\hline ATCC $31749 \Delta$ nifR & ATCC 31749 mutant with gene knockout of nifR & This study \\
\hline ATCC $31749 \triangle p p \times 1$ & ATCC 31749 mutant with gene knockout of ppx1 (AGRO_2774) & This study \\
\hline ATCC $31749 \triangle p p \times 2$ & ATCC 31749 mutant with gene knockout of ppx2 (AGRO_2552) & This study \\
\hline ATCC 31749 $\Delta r p o N$ & ATCC 31749 mutant with gene knockout of rpoN & This study \\
\hline ATCC 31749 $\Delta r r p P$ & ATCC 31749 mutant with gene knockout of $r r p P$ & This study \\
\hline 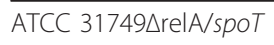 & ATCC 31749 mutant with gene knockout of AGRO_1497 & This study \\
\hline ATCC $31749 \triangle 0033$ & ATCC 31749 mutant with gene knockout of AGRO_0033 & This study \\
\hline ATCC $31749 \triangle 0636$ & ATCC 31749 mutant with gene knockout of AGRO_0636 & This study \\
\hline ATCC $31749 \triangle 1729$ & ATCC 31749 mutant with gene knockout of AGRO_1729 & This study \\
\hline ATCC $31749 \triangle 3967$ & ATCC 31749 mutant with gene knockout of AGRO_3967 & This study \\
\hline DH5a/pUCP30T & E. coli strain containing suicide vector & H. Schweitzer \\
\hline pGEM-T easy & Vector used for cloning internal gene fragments & Promega \\
\hline pUCP30T-nifR & Gene knockout plasmid for interruption of nifR & This study \\
\hline pUCP30T-ppx1 & Gene knockout plasmid for interruption of ppx1 (AGRO_2774) & This study \\
\hline pUCP30T-ppx2 & Gene knockout plasmid for interruption of ppx2 (AGRO_2552) & This study \\
\hline pUCP30T-rpoN & Gene knockout plasmid for interruption of rpoN & This study \\
\hline pUCP30T-rrpP & Gene knockout plasmid for interruption of $r r p P$ & This study \\
\hline pUCP30T-spoT & Gene knockout plasmid for interruption of spoT & This study \\
\hline pUCP30T-0033 & Gene knockout plasmid for interruption of AGRO_0033 & This study \\
\hline PUCP30T-0636 & Gene knockout plasmid for interruption of AGRO_0636 & This study \\
\hline pUCP30T-1729 & Gene knockout plasmid for interruption of AGRO_1729 & This study \\
\hline pUCP30T-3967 & Gene knockout plasmid for interruption of AGRO_3967 & This study \\
\hline
\end{tabular}

and Agilent's Feature Extraction 10.5 Image Analysis Software was used to measure the signal intensities.

\section{Microarray data analysis}

The mean local background signal intensity was subtracted from the mean signal intensity for each probe spot on the microarray. The background-subtracted signals were then normalized using a lowess normalization to account for dye-labeling bias. The lowessnormalized data was uploaded into GeneSpring GX 10, and $75^{\text {th }}$ percentile normalization was applied. The normalized data was then analyzed for statistically significant changes in gene expression using a paired $T$ test with a p-value cutoff of 0.05. Statistically significant genes with more than a 2 -fold change in gene expression were manually categorized according to function.

\section{Curdlan synthesis with knockout mutants}

For each mutant, the seed culture was inoculated into two flasks containing $150 \mathrm{~mL}$ LB media (100x dilution). The cultures were grown at $30^{\circ} \mathrm{C}$ and $250 \mathrm{rpm}$ until stationary phase (approximately 12 hours, $\mathrm{OD}_{600}>1.5$ ) or the late exponential phase $\left(1.3<\mathrm{OD}_{600}<1.5\right)$ was reached. The cells were collected by centrifugation $\left(3,000 \times \mathrm{g}\right.$ and $4^{\circ} \mathrm{C}$ for $\left.10 \mathrm{~min}\right)$. After one wash step with $10 \%$ glycerol, the cell pellets were resuspended in nitrogen-free minimal media, containing $40 \mathrm{~g} / \mathrm{L}$ sucrose, $0.5 \mathrm{~g} / \mathrm{L} \mathrm{KH}_{2} \mathrm{PO}_{4}, 0.5 \mathrm{~g} / \mathrm{L} \mathrm{MgSO}_{4} \cdot 7 \mathrm{H}_{2} \mathrm{O}$, and $10 \mathrm{~mL} / \mathrm{L}$ of trace element solution [30] (autoclaved separately), $\mathrm{pH}$ 7. Nitrogen-free cell cultures were incubated at $30^{\circ} \mathrm{C}$ and $250 \mathrm{rpm}$ with sampling at 24 hour intervals. PolyP levels in exponential phase cultures were measured as described previously [32].

\section{Quantitative RT-PCR}

Samples (3 mL) were taken at 24, 72, and 120 hours after the shift to nitrogen-free media. RNA was isolated as described above. DNase treated RNA $(2 \mu \mathrm{g})$ was converted to cDNA using Invitrogen's Superscript III first-strand synthesis system. Gene expression was analyzed using Applied Biosystems' SYBR green PCR master mix and 7300 Real-Time PCR System. A constitutively expressed sigma factor, $r p o D$, was used for normalization, and primers for $c r d S$ and $r p o D$ measurement are listed in Table 4 . The $2-\Delta \Delta C_{\mathrm{T}}$ method 
Table 4 Primers used in this study.

\begin{tabular}{|c|c|c|}
\hline Target Gene & Forward/Reverse & Primer Sequence \\
\hline \multicolumn{3}{|l|}{ Internal gene fragment } \\
\hline \multirow[t]{2}{*}{ nifR } & $\mathrm{F}$ & 5'- GCAAGCTTCCCAATCCGCCCGTCCCTC - 3' \\
\hline & $\mathrm{R}$ & 5'- CAGAGCTCGAAATGGTCGCCAGCCGC - 3' \\
\hline \multirow[t]{2}{*}{ ppx1 (AGRO_2774) } & $\mathrm{F}$ & 5'- CTAAGCTTGCAGCGAGGTCCAGTGGGTG - 3' \\
\hline & $\mathrm{R}$ & 5'- CAGAGCTCCAGTTCCGGGTCGTCGATG - 3' \\
\hline \multirow[t]{2}{*}{ ppx2 (AGRO_2552) } & $\mathrm{F}$ & 5'- CTAAGCTTGCTCCAATCCGCCAGTTCGC - 3' \\
\hline & $\mathrm{R}$ & 5'- CAGAGCTCGGTCGGTGGTACATGGCGAA - 3' \\
\hline \multirow[t]{2}{*}{$r p o N$} & $\mathrm{~F}$ & 5'- GCAAGCTTCATCGAATCGTAACCCGCG - 3' \\
\hline & $\mathrm{R}$ & 5'- GCGAGCTCAGCGACCACCTCAATCAG - 3' \\
\hline \multirow[t]{2}{*}{$\operatorname{rrp} P$} & $\mathrm{~F}$ & 5'- GTAAGCTTGATCAGCGCCGTCACGACAAG - 3' \\
\hline & $\mathrm{R}$ & 5'- CAGAGCTCCCGCGCAACCCTGCTACCAT - 3' \\
\hline \multirow[t]{2}{*}{ relA/spoT (AGRO_1497) } & $\mathrm{F}$ & 5'- GTAAGCTTGTATGCTCCAGGAACTCTTC - 3' \\
\hline & $\mathrm{R}$ & 5'- GAGAGCTCCGTCAAGGGACGTCAGAAAA - 3' \\
\hline \multirow[t]{2}{*}{ AGRO_0033 } & $\mathrm{F}$ & 5'- CGTGACAAGCTITTCATAGATGCAGGCAACGTC - 3' \\
\hline & $\bar{R}$ & 5'- GAACGTGAGCTCCTGGTGGGCGGATGATTGT - 3' \\
\hline \multirow[t]{2}{*}{ AGRO_0636 } & $\mathrm{F}$ & 5'- CCTAGAAAGCTTCACAATAGTCGCTGCCAAGT - 3' \\
\hline & $R$ & 5'- CCTAGAGAGCTCGCTTGTCTCGTGACGCTCATT - 3' \\
\hline \multirow[t]{2}{*}{ AGRO_1729 } & $\mathrm{F}$ & 5'- CGTGACAAGCTTCGGGATGGCAATGGTCTCGT - 3' \\
\hline & $\mathrm{R}$ & 5'- CTTGTAGAGCTCCTGGCGTTGATACCCATGCT - 3' \\
\hline \multirow[t]{2}{*}{ AGRO_3967 } & $\mathrm{F}$ & 5'- GAGTGTAAGCTITCCAGCACATAATAGGGCGA - 3' \\
\hline & $R$ & 5'- CTTGGAGAGCTCGACACTTGCGGTTGTCATCG - 3' \\
\hline \multicolumn{3}{|c|}{ Gene knockout confirmation } \\
\hline $\mathrm{GM}^{\mathrm{R}}$ & $\mathrm{F}$ & 5'- GATGCCCATACTTGAGCCACCTAAC - 3' \\
\hline nifR & $\mathrm{R}$ & 5'- AAGATCATCATTTGCCTCTTCCGGAG - 3' \\
\hline ppx1 (AGRO_2774) & $\mathrm{R}$ & 5'- GCGAAGCGATCCCGTCGTGGCAAGA - 3' \\
\hline ppx2 (AGRO_2552) & $\mathrm{R}$ & 5'- GACTCGATCAGAAGCACAGGGGC - 3' \\
\hline$r p o N$ & $\mathrm{R}$ & 5'- ATGACGCATTTCGAGCTGACGCAGTT - 3' \\
\hline $\operatorname{rrp} P$ & $\mathrm{R}$ & 5'- ATGCGGTGTCCTGTCCGTGGTTTA - 3' \\
\hline relA_spoT (AGRO_1497) & $\mathrm{R}$ & 5'- CGCTITCTIITTGTGGAATATCGCA - 3' \\
\hline AGRO_0033 & $\mathrm{R}$ & 5'- GTCGTGTTTCAAGTTTCAGGGTCCG - 3' \\
\hline AGRO_0636 & $\mathrm{R}$ & 5'- GATGTTGGGTCTITGGAAAAAACCG - 3' \\
\hline AGRO_1729 & $\mathrm{R}$ & 5'- ATGAATGGTGAAAAGCGGGGGAGC - 3' \\
\hline AGRO_3967 & $\mathrm{R}$ & 5'- GGTGAATCGCAGATGATGGAATTGAT - 3' \\
\hline \multicolumn{3}{|l|}{ Quantitative RT-PCR } \\
\hline \multirow[t]{2}{*}{ crds } & $\mathrm{F}$ & 5'- ATCCAATTCAGCACAATCTCG -3' \\
\hline & $\mathrm{R}$ & 5'- ACATATCCCCTITCCATCAG -3' \\
\hline \multirow[t]{2}{*}{$r p o D$} & $\mathrm{~F}$ & 5'- GGAAATCCAGAACCTCTCCAC -3' \\
\hline & $\mathrm{R}$ & 5'- GAACTTGTAACCACGGCGATA -3' \\
\hline
\end{tabular}

Restriction enzyme sites are underlined. For gene knockout confirmation, the primer homologous to the gentamicin resistance gene $\left(G M^{R}\right)$ was used along with a primer homologous to the sequence upstream of the target gene

was applied to analyze the change in gene expression between the wild type and knockout strains, $\Delta p p \times 2$ and $\Delta r e l A / s p o T[33]$.

\section{Abbreviations}

AA: Amino acid; ADP: Adenosine diphosphate; Alg44: Putative alginate biosynthesis protein; ATP: Adenosine triphosphate; BcsA: Cellulose synthase;
$\mathrm{Ca}^{2+}$ : Calcium ion; c-di-GMP: bis- $\left(3^{\prime}, 5^{\prime}\right)$-cyclic-dimeric-guanosine monophosphate; cfu: Colony forming units; DGC: Diguanylate cyclase; EPS: Exopolysaccharide; FleQ: Transcriptional regulator of flagella and PEL synthesis; GDP: Guanosine diphosphate; GlnB: Pll protein; GlnD: Nitrogen sensor protein; GInK: PII protein; GMP: Guanosine monophosphate; GppA: pppGpp phosphohydrolase; GTP: Guanosine triphosphate; $\mathrm{H}^{+}$: Proton; NDK: Nucleoside diphosphate kinase; NtrB: Histidine kinase/phosphatase; NtrC: Nitrogen assimilation regulatory protein; PDE: Phosphodiesterase; PelE: PEL exopolysaccharide biosynthesis protein; polyP: Polyphosphate; Pi: Phosphate; 
PPase: Pyrophosphatase; ppGpp: Guanosine tetraphosphate; PPi: Pyrophosphate; PPK: Polyphosphate kinase; pppGpp: Guanosine pentaphosphate; PPX: Exopolyphosphatase; RelA: (p)ppGpp synthetase; RNAP: RNA polymerase; RpoN: Sigma factor 54; SpoT: ppGpp pyrophosphohydrolase/(p)ppGpp synthetase

\section{Acknowledgements}

We thank Dr. Herbert Schweizer (Colorado State University) for supplying the suicide vector, pUCP30T. This work was funded by National Science Foundation (BES 0455193) and the American Cancer Society.

\section{Authors' contributions}

AMR carried out all experiments presented in this work, with the exception of the CDNA library preparation and microarray hybridization which was performed by Gene Logic, Inc. AMR and RRC participated in the design of the study, analysis of the results, and drafting of the manuscript. All authors read and approved the final manuscript.

\section{Competing interests}

The authors declare that they have no competing interests.

Received: 22 December 2011 Accepted: 3 February 2012

Published: 3 February 2012

\section{References}

1. Sutherland W: Novel and established applications of microbial polysaccharides. Trends Biotechnol 1998, 16:41-46.

2. Kumar AS, Mody K, Jha B: Bacterial exopolysaccharides - a perception. $J$ Basic Microbiol 2007, 47:103-117.

3. McIntosh M, Stone BA, Stanisich VA: Curdlan and other bacterial (1à3)- $\beta$ D-glucans. Appl Microbiol Biotechnol 2005, 68:163-173.

4. Stasinopoulos SJ, Fisher PR, Stone BA, Stanisich VA: Detection of two loci involved in (1à3)- $\beta$-glucan (curdlan) biosynthesis by Agrobacterium sp. ATCC31749, and comparative sequence analysis of the putative curdlan synthase gene. Glycobiology 1999, 9:31-41.

5. Leigh JA, Dodsworth JA: Nitrogen regulation in bacteria and archaea. Annu Rev Microbiol 2007, 61:349-377.

6. Ruffing AM, Castro-Melchor M, Hu W-S, Chen RR: Genome sequence of the curdlan-producing Agrobacterium sp. strain ATCC 31749. J Bacteriol 2011, 193:4294-4295.

7. Seufferheld M, Vieira MCF, Ruiz FA, Rodrigues CO, Moreno SNJ, Docampo R: Identification of organelles in bacteria similar to acidocalcisomes of unicellular eukaryotes. J Biol Chem 2003, 278:29971-29978.

8. Docampo R, de Souza W, Miranda K, Rohloff P, Moreno SNJ: Acidocalcisomes - conserved from bacteria to man. Nature Reviews Microbiology 2005, 3:251-261.

9. Kornberg A: Inorganic polyphosphate: Toward making a forgotten polymer unforgettable. J Bacteriol 1995, 177:491-496.

10. Kim H-Y, Schlictman D, Shankar S, Xie Z, Chakrabarty AM, Kornberg A: Alginate, inorganic polyphosphate, GTP and ppGpp synthesis coregulated in Pseudomonas aeruginosa: implications for stationary phase survival and synthesis of RNA/DNA precursors. Mol Microbiol 1998, 27:717-725.

11. Ault-Riche D, Fraley CD, Tzeng C-M, Kornberg A: Novel assay reveals multiple pathways regulating stress-induced accumulations of inorganic polyphosphate in Escherichia coli. J Bacteriol 1998, 180:1841-1847.

12. Rao NN, Gómez-García MR, Kornberg A: Inorganic Polyphosphate: Essential for Growth and Survival. Annu Rev Biochem 2009, 78:605-647.

13. Hengge R: Principles of c-di-GMP signalling in bacteria. Nat Rev Microbiol 2009, 7:263-273.

14. Rehm BHA, Valla S: Bacterial alginates: biosynthesis and applications. App/ Microbiol Biotechnol 1997, 48:281-288.

15. Kim MK, Lee IY, Ko JH, Rhee YH, Park YH: Higher intracellular levels of uridinemonophosphate under nitrogen-limited conditions enhance metabolic flux of curdlan synthesis in Agrobacterium species. Biotechnol Bioeng 1999, 62:317-323.

16. Magnusson LU, Farewell A, Nyström T: ppGpp: a global regulator in Escherichia coli. Trends Microbiol 2005, 13:236-242.

17. Zheng Z-Y, Lee J, Zhan X, Shi Z, Wang L, Zhu L, Wu J-R, Lin C: Effect of metabolic structures and energy requirements on curdlan production by Alcaligenes faecalis. Biotechnol Bioprocess Eng 2007, 12:359-365.
18. Seufferheld MJ, Alvarez HM, Farias ME: Role of polyphosphates in microbial adaptation to extreme environments. Appl Environ Microbiol 2008, 74:5867-5874.

19. Kuroda A, Murphy H, Cashel M, Kornberg A: Guanosine tetra- and pentaphosphate promote accumulation of inorganic polyphopshate in Escherichia coli. J Biol Chem 1997, 272:21240-21243.

20. Lee J-H, Lee I-Y, Kim M-K, Park Y-H: Optimal pH control of batch processes for production of curdlan by Agrobacterium species. J Ind Microbiol Biotechnol 1999, 23:143-148.

21. Srivatsan A, Wang JD: Control of bacterial transcription, translation and replication by (p)ppGpp. Curr Opin Microbiol 2008, 11:100-105.

22. Crooke E, Akiyama M, Rao NN, Kornberg A: Genetically altered levels of inorganic polyphosphate in Escherichia coli. J Biol Chem 1994, 269:6290-6295.

23. Gentry DR, Hernandez VJ, Nguyen LH, Jensen DB, Cashel M: Synthesis of the stationary-phase sigma factor sigma $\mathrm{s}$ is positively regulated by ppGpp. J Bacteriol 1993, 175:7982-7989.

24. Foster-Hartnett D, Cullen PJ, Monika EM, Kranz RG: A new type of NtrC transcriptional activator. J Bacteriol 1994, 176:6175-6187.

25. Foster-Hartnett D, Cullen PJ, Gabbert KK, Kranz RG: Sequence, genetic, and lacZ fusion analysis of a nifR3-ntrB-ntrC operon in Rhodobacter capsulatus. Mol Microbiol 1993, 8:903-914.

26. Wells DH, Long SR: The Sinorhizobium meliloti stringent response affects multiple aspects of symbiosis. Mol Microbiol 2002, 43:1115-1127.

27. van Delden C, Comte R, Bally M: Stringent response activates quorum sensing and modulates cell density-dependent gene expression in Pseudomonas aeruginosa. J Bacteriol 2001, 183:5376-5384.

28. Quiñones B, Dulla G, Lindow SE: Quorum sensing regulates exopolysaccharide production, motility, and virulence in Pseudomonas syringae. Mol Plant Microbe Interact 2005, 18:682-693.

29. Yuan Z-C, Liu P, Saenkham P, Kerr K, Nester EW: Transcriptome profiling and functional analysis of Agrobacterium tumefaciens reveals a general conserved response to acidic conditions ( $\mathrm{pH}$ 5.5) and a complex acidmediated signaling involved in Agrobacterium-plant interactions. Journal of Bacteriology 2008, 190:494-507.

30. Kim MK, Lee IY, Lee JH, Kim KT, Rhee YH, Park YH: Residual phosphate concentration under nitrogen-limiting conditions regulates curdlan production in Agrobacterium species. I Ind Microbiol Biotechnol 2000, 25:180-183.

31. Srienc F, Arnold B, Bailey JE: Characterization of intracellular accumulation of poly- $\beta$-hydroxybutyrate (PHB) in individual cells of Alcaligenes eutrophus H16 by flow cytometry. Biotechnol Bioeng 1984, 26:982-987.

32. Aschar-Sobbi R, Abramov A, Diao C, Kargacin M, Kargacin G, French R, Pavlov E: High sensitivity, quantitative measurements of polyphosphate using a new DAPI-based approach. J Fluoresc 2008, 18:859-866.

33. Livak KJ, Schmittgen TD: Analysis of relative gene expression data using real-time quantitative PCR and the 2- $\Delta \Delta C T$ method. Methods 2001, 25:402-408.

doi:10.1186/1475-2859-11-17

Cite this article as: Ruffing and Chen: Transcriptome profiling of a curdlan-producing Agrobacterium reveals conserved regulatory mechanisms of exopolysaccharide biosynthesis. Microbial Cell Factories 2012 11:17.

\section{Submit your next manuscript to BioMed Central and take full advantage of:}

- Convenient online submission

- Thorough peer review

- No space constraints or color figure charges

- Immediate publication on acceptance

- Inclusion in PubMed, CAS, Scopus and Google Scholar

- Research which is freely available for redistribution 